\title{
Reply to the response by Crosby et al. to: "Glenosphere disengagement in a reverse total shoulder arthroplasty with a non-Morse taper design"
}

\author{
Corey Fuller ${ }^{1}$ Eric Lim ${ }^{2}$
}

Received: 15 March 2015 / Accepted: 17 March 2015 / Published online: 24 April 2015

(C) SICOT aisbl (outside the USA) 2015

We appreciate Exactech's whole-hearted defense of their product design [1]. We do not dispute Exactech's explanation and want to be clear that we do not blame these complications on their product design. Our goal was not to disparage their product but to report on our apparently unique complications and present our musings on why they occurred [2]. It should be noted that the vast majority of the patients who received this implant (including those mentioned in the article) are now doing well. That being said, in our experience, implanting the glenosphere onto the glenoid baseplate was consistently the most difficult part of this surgery. Hopefully, others can learn from our challenges and our experience will be an isolated one.

\section{References}

1. Cosby L et al (2015) Response to: Fuller et al., "Glenosphere disengagement in a reverse total shoulder arthroplasty with a non-Morse taper design". Int Orthop. doi:10.1007/s00264-015-2756-0

2. Fuller CB et al (2015) Glenosphere disengagement in a reverse total shoulder arthroplasty with a non-Morse tapered design. Int Orthop 39(2):305-308
Corey Fuller

cbfuller@llu.edu

1 Department of Orthopedic Surgery, Loma Linda University, Loma Linda, CA, USA

2 Department of Orthopedic Surgery, Loma Linda Veterans Hospital, Loma Linda, CA, USA 